\title{
Analyzing Power Measurements in Elastic proton-proton Scattering at 24 and $400 \mathrm{GeV}$
}

\author{
Richard A. Phelps \\ Department of Physics, The University of Michigan \\ Ann Arbor, Michigan 48109-1120 USA
}

\begin{abstract}
Recent measurements of the analyzing power, $\mathrm{A}$, in protonproton elastic scattering at $24 \mathrm{GeV} / \mathrm{c}$ out to $\mathrm{P}_{\perp}^{2}=7.1(\mathrm{GeV} / \mathrm{c})^{2}$ at the AGS clearly show that $\mathrm{A}$ is nonzero and increasing at high $\mathrm{P}_{\perp}^{2}$. An experiment to extend these measurements to $400 \mathrm{GeV}$ and $3 \mathrm{TeV}$ out to $\mathrm{P}_{\perp}^{2}=8(\mathrm{GeV} / \mathrm{c})^{2}$ at the UNK accelerator in Protvino, USSR is also discussed.
\end{abstract}

I will report on recent results and future plans of an experimental program for measuring spin parameters in pp elastic scattering. This particular program began at the Argonne $\mathrm{ZGS}^{[1,2]}$ in the 1970's, continued at the Brookhaven $\mathrm{AGS}^{[3-5]}$ in the 1980's, and will continue at UNK in Protvino, USSR in the 1990's.

The focus here will be on the analyzing power, A, for pp elastic scattering at high $\mathrm{P}_{\perp}^{2}$. To measure $\mathrm{A}$, one needs either a polarized beam or polarized target. The measurements discussed here use a polarized target with the polarization transverse to the scattering plane.

The analyzing power measures the change in the scattering rate when the polarization is reversed:

$$
\mathrm{A}=\frac{1}{\mathrm{P}}\left[\frac{\mathrm{d} \sigma(\uparrow)-\mathrm{d} \sigma(\downarrow)}{\mathrm{d} \sigma(\uparrow)+\mathrm{d} \sigma(\downarrow)}\right]
$$

The target polarization direction is either $\uparrow$ or $\downarrow$ and its magnitude is $P$.

A detailed description of the experimental setup at Brookhaven has been given elsewhere ${ }^{[5]}$, hence only a brief outline will be given here. This experiment was a collaboration of the University of Michigan, Brookhaven, Texas A \& M, TRIUMF, and King Fahd University in Saudi Arabia.

Our target material was radiation doped frozen ammonia $\left(\mathrm{NH}_{3}\right)$. The target itself was a $1 \mathrm{~K}^{4} \mathrm{He}$ evaporation refrigerator with a $5 \mathrm{~T}$ superconducting split coil magnet supplied by Oxford Instruments. Polarization of the free hydrogen was achieved using the Dynamic Nuclear Polarization method ${ }^{[6,7]}$. With this new target we could take about four times the beam intensity $\left(2 \times 10^{11}\right.$ protons/pulse) of previous runs and the average target polarization was $85 \%$ (vs. $50 \%$ for our previous target). These effects combined to improve our errors in A by a factor of about three over what we could achieve with our previous target. 


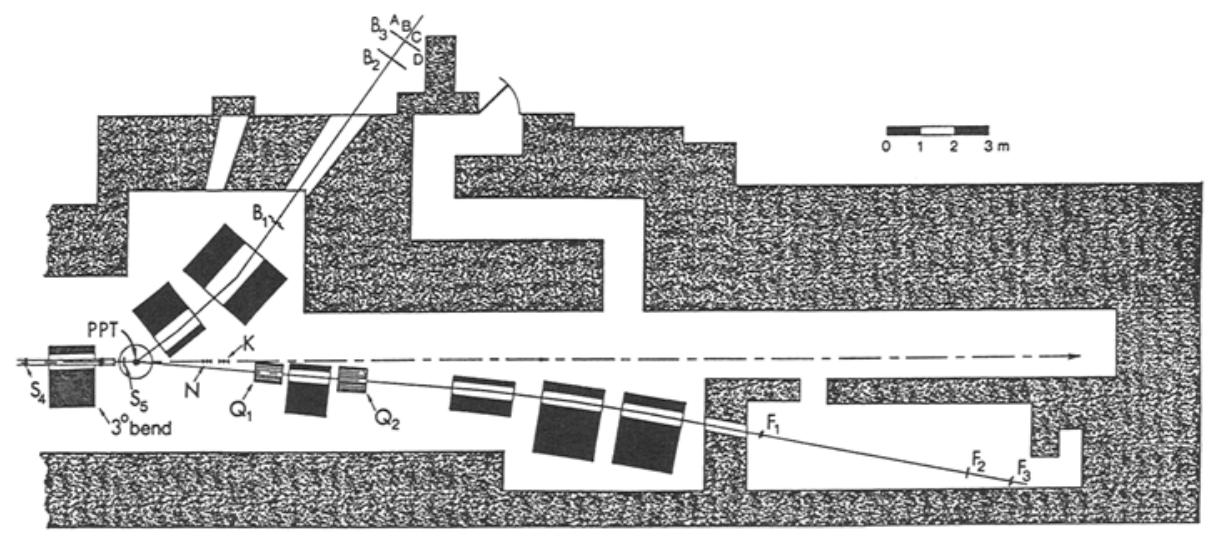

Fig. 1 Diagram of the BNL experiment. The N and $\mathrm{K}$ counters were intensity monitors, while the $S_{4}$ and $S_{5}$ segmented wire ion chambers monitored the beam's position, size and angle.

The double arm spectrometer is shown in Fig. 1. Elastic events are selected by collimation, bending magnets, and a 6 fold coincidence between 3 forward $(F)$ and 3 recoil (B) hodoscopes in the spectrometer. Other relevant hardware were two focusing quadrupoles $\left(Q_{1}\right.$ and $\left.Q_{2}\right)$ which doubled our forward arm acceptance to about $10^{-3}$ sr and a $3^{\circ}$ bending magnet just before the PPT which allowed us to go to higher $\mathrm{P}_{\perp}^{2}$ without drastically altering our spectrometer geometry.

Elastic cross sections drop sharply with increasing $\mathrm{P}_{\perp}^{2}$; for example, $(\mathrm{d} \sigma / \mathrm{d} \Omega)_{\mathrm{cm}}$ is $1.6 \mathrm{nb} / \mathrm{sr}$ at $\mathrm{P}_{\perp}^{2}=6(\mathrm{GeV} / \mathrm{c})^{2}$, and it drops by another factor of three at $\mathrm{P}_{\perp}^{2}=7(\mathrm{GeV} / \mathrm{c})^{2}$. With such small cross sections, we needed the luminosity of $2 \times 10^{34} \mathrm{~cm}^{-2} \mathrm{~s}^{-1}$ to get good statistics.

The new data in the $\mathrm{P}_{\perp}^{2}$ range of 3.2 to $7.1(\mathrm{GeV} / \mathrm{c})^{2}$, is given in Fig. 2 along with data from our earlier $28 \mathrm{GeV} / \mathrm{c} \mathrm{run}^{[4]}$ and $24 \mathrm{GeV} / \mathrm{c}$ data from CERN. ${ }^{\left[{ }^{[9]}\right.}$ The errors shown include statistical errors as well as a $3 \%$ relative error in the target polarization. The data was corrected for accidental coincidences and background from scattering off bound protons in $\mathrm{NH}_{3}$. This was measured by performing a run where our target material was teflon $\left(\mathrm{CF}_{2}\right)$ which contains no hydrogen.

The data clearly indicates that the analyzing power is nonzero and increasing at high $\mathrm{P}_{\perp}^{2}$. Calculations of $\mathrm{A}$ based on perturbative $\mathrm{QCD}$ (where quark helicity is conserved) predict that A should be zero. This data certainly shows that even at these energies and $P_{\perp}^{2}$ 's, non-perturbative effects play a strong role, and present a challenge to any theorist searching for an explanation. While a satisfactory answer is being sought, we plan to forge ahead to see if this effect persists at even higher energies and $\mathrm{P}_{\perp}^{2}$. 
Thus, we are presently designing and constructing an experiment to measure $\mathrm{A}$ for pp elastic scattering in the $400 \mathrm{GeV}$ to $3 \mathrm{TeV}$ range, and for $\mathrm{P}_{\perp}^{2}$ of up to $8(\mathrm{GeV} / \mathrm{c})^{2}$. This experiment (designated NEPTUN-A) will take place at the currently under construction UNK accelerator in Protvino, USSR. NEPTUN-A is a collaboration of IHEP in Protvino, JINR in Dubna, MIT, and the University of Michigan.

The NEPTUN-A experimental setup is shown in Fig. 3. It contains a 55 meter long recoil spectrometer with a $12^{\circ}$ vertical bend for momentum selection. The planned recoil momentum resolution for this arm is $0.1 \%$, and the planned recoil angle resolution is $0.3 \mathrm{mrad}$. Minimal information will be needed from the forward arm to separate out the elastic signal; we will detect the forward particle with two forward hodoscopes. This momentum and angle resolution will be achieved using the hodoscopes $\mathrm{H}_{\mathbf{i}}$ at the beginning and end of the

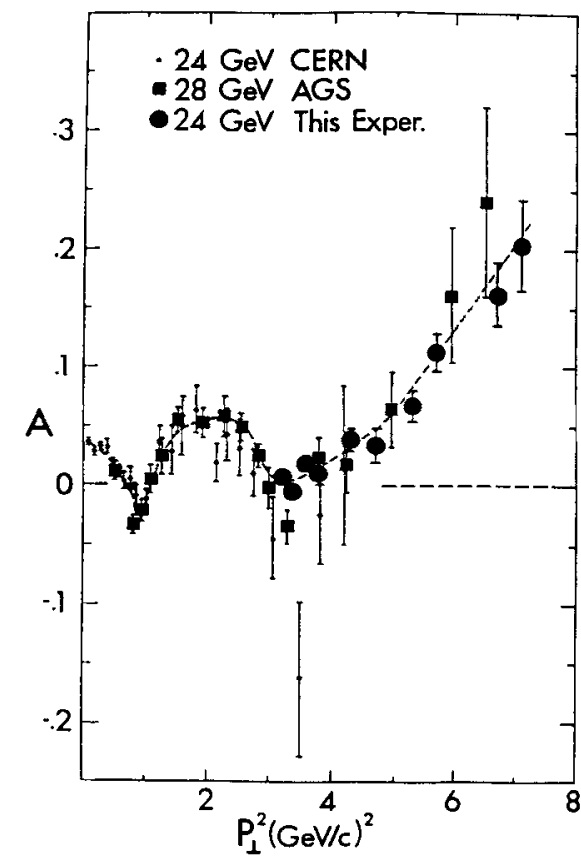

Fig. 2 The analyzing power A as a function of momentum transfer squared $\mathrm{P}_{\perp}^{2}$. Other data at $28^{[4]}$ and $24^{[9]} \mathrm{GeV} / \mathrm{c}$ are also shown. The dashed curve is a hand-drawn curve to guide the eye. recoil arm and multi-wire proportional chambers $\mathrm{W}_{\mathrm{i}}$ for good particle track position resolution. The information from these detectors will be supplemented with a time-of-flight measurement between the hodoscopes and also by threshold Cherenkov counters for velocity selection. With this extra information, we should be able to distinguish between protons, kaons, and pions. The spin asymmetry in inclusive reactions can then be studied.

The internal gas target will be an ultra-cold spin polarized atomic hydrogen target being built at the University of Michigan. We hope to achieve a target thickness of $10^{14} / \mathrm{cm}^{2}$ and UNK should eventually have an intensity of $10^{19}$ protons per sec passing through our target; this would give a luminosity of $10^{33}$ $\mathrm{cm}^{-1} \mathrm{sec}^{-1}$. Our predicted errors in a measurement of $\mathrm{A}$ range from $0.2 \%$ for $\mathrm{P}_{\perp}^{2}=2(\mathrm{GeV} / \mathrm{c})^{2}$ to $3.8 \%$ for $\mathrm{P}_{\perp}^{2}=8(\mathrm{GeV} / \mathrm{c})^{2}$, based on our approved hours of running. Our first run at $400 \mathrm{GeV} / \mathrm{c}$ should be in October 1993 . 




Fig. 3 Diagram of the NEPTUN-A experiment (Top View). The $\mathrm{H}_{\mathrm{i}}$ 's are scintillation-counter hodoscopes, the $\mathrm{W}_{\mathrm{i}}$ 's are wire chambers and the $\mathrm{C}_{\mathrm{i}}$ 's are threshold cherenkov counters. Magnets include pairs of focusing quadrupoles $\left(Q_{i}\right.$ 's) and dipole bending magnets (M6 vertical and all others horizontal).

This research was supported by a grant from the U.S. Department of Energy.

\section{REFERENCES}

1. D. G. Crabb et al., Phys. Rev. Lett. 41, 1257 (1978); J. R. O'Fallon et al., Phys. Rev. Lett. 39, 733 (1977); K. Abe et al., Phys. Lett. 63B, 239 (1976).

2. E. A. Crosbie et al., Phys. Rev. D23, 600 (1981).

3. K. A. Brown et al., Phys. Rev. D31, 3017 (1985); G. R. Court et al., Phys. Rev. Lett. 57, 507 (1986); D. G. Crabb et al., Phys. Rev. Lett. 60, 2351 (1988); F. Z. Khiari et al., Phys. Rev. D39, 45 (1989).

4. P. R. Cameron et al., Phys. Rev. D32, 3070 (1985); P. H. Hansen et al., Phys. Rev. Lett. 50, 802 (1983); D. C. Peaslee et al., Phys. Rev. Lett. 51, 2359 (1983).

5. D. G. Crabb et al., Phys. Rev. Lett. 65, 3241 (1990).

6. A. Abragam and N. Goldman, Rep. Prog. Phys. 41, 395 (1978).

7. C. D. Jeffries, "Dynamic Nuclear Orientation" John Wiley \& Sons (New York 1963).

8. D. G. Crabb et al., Phys. Rev. Lett. 64f, 2627 (1990).

9. J. Antille et al., Nucl. Phys. B185, 1 (1981). 